\title{
"Tante cose venivano pubblicate ma hanno dovuto aspettare un altro momento": la literatura latinoamericana y sus re-traducciones
}

\section{Introducción}

Los estudios sobre la recepción de la literatura latinoamericana en Italia se han centrado, por lo general, en el análisis de la cantidad de títulos publicados o en la recepción de obras y autores específicos. El ámbito crítico todavía carece de una visión de conjunto en la que el objeto libro se analice en relación con las dinámicas editoriales. A no ser por el trabajo de Stefano Tedeschi All'inseguimento dell'ultima utopia, donde se delinea una historia de la recepción a través del análisis de traducciones y reseñas que el autor interpreta a partir de una perspectiva socio-literaria, los estudios de recepción sobre la difusión de la literatura latinoamericana en Italia no cuentan con un estudio que tome nota de cómo evolucionaron las dinámicas internas al ámbito literario con respecto a las dinámicas editoriales y culturales italianas de 1950 en adelante.

Este tipo de investigación supone repensar el análisis de la recepción a partir del estudio de las modalidades a través de las que se cumple el encuentro entre "mundo del texto" y "mundo del lector" (Ricoeur 228-263) tanto desde un punto de vista socio-literario como desde un punto de vista editorial, incluyendo todas esas praxis que se dan alrededor de un libro y que, cuando se identifican, permiten una visión más amplia del fenómeno receptivo y facilitan la identificación de las modalidades de acceso, incluyendo "los espacios, los gestos y las costumbres” (Chartier 17), que la comunidad de lectores -en este caso italianos- ha utilizado para leer textos procedentes de América Latina.

Tomando como punto de referencia al estudio de Chartier L'ordre du livre, un texto puede aparecer en un contexto cultural en diferentes épocas y bajo diversas modalidades (24-30). Esto comportará una aproximación diferente de la comunidad lectora hacia un determinado libro y, en consecuencia, también un acercamiento distinto a su recepción y a su inclusión en el campo cultural. Sin embargo, la primera recepción no es la del público, sino que se da en primer lugar en los ámbitos editoriales -que supuestamente deciden si apoyar o no

Sara Carini, Università Cattolica del Sacro Cuore

Ә Open Access. (C) 2020 Sara Carini, published by De Gruyter. (c) BY-NC-ND This work is licensed under a Creative Commons Attribution-NonCommercial-NoDerivatives 4.0 International License.

https://doi.org/10.1515/9783110673678-011 
una traducción y establecen el aspecto que tendrá un libro- y, en segundo lugar, en el ámbito cultural especializado, donde trabajan profesionales de la cultura más o menos vinculados con el mundo editorial y literario.

Como afirma Alberto Cadioli, el estudio de la relación entre editoriales y libros editados ayuda a comprender cómo se ha desarrollado la oferta literaria porque permite comprender las razones que conforman la base de una acción editorial (11). Pero la oferta propuesta por una editorial no puede moverse libre dentro del campo cultural y literario, se encuentra bajo el control de las dinámicas que económica, política y literariamente definen las modas y las pautas de la oferta literaria y de la cultural global. A nivel de recepción, aproximarnos al análisis de los fenómenos culturales que se desarrollan alrededor del objeto libro permite valorar los modos de inserción aplicados a un libro en traducción y permite, cuando esto sea posible, considerar la cantidad de recursos que se han invertido en la edición de un libro y también la postura que mantuvieron los medios culturales y literarios. De hecho, conocer el grado de disponibilidad y el tipo de competencias que un campo cultural puso a disposición de una literatura extranjera es el primer dato de interés a la hora de comprender cómo se ha desarrollado su difusión en un campo literario y cultural diferente al original, más allá de la consideración de la simple cuota "virtual” de lectores que el libro puede alcanzar por haber entrado en un catálogo editorial extranjero.

\section{Casos de estudio: Farabeuf y Paradiso en los siglos XX y XXI}

Siguiendo este enfoque, lo que proponemos en este estudio es el análisis de las reseñas a dos ediciones diferentes de las novelas Farabeuf, de Salvador Elizondo, y Paradiso, de José Lezama Lima, editadas en 1970 y 1971 respectivamente por las editoriales il Saggiatore y Feltrinelli y, en 2018 y 2016, por las editoriales LiberAria y SUR. Nuestro objetivo es identificar las dinámicas que entraron en juego a la hora de introducir un texto al público. Con esta aproximación queremos delinear las características del ámbito de recepción en el que tuvieron que insertarse los textos latinoamericanos en traducción y, en particular, nos centraremos en las reseñas que se produjeron tras la primera y la última edición de ambas novelas porque nos interesa comparar la primera recepción, que se dio en los años del primer boom de la narrativa latinoamericana en Italia, con la última, que, en cambio, se dio en un nuevo contexto de 
referencias literarias, en el que predominan la voz de los narradores nacidos alrededor de los 70 y el mito de Roberto Bolaño.

\subsection{Premisas necesarias: el campo literario italiano y la literatura latinoamericana}

La recepción de los latinoamericanos en Italia fue bastante peculiar. Para delinearlo de forma rápida nos parecen esclarecedoras las palabras con las que Italo Calvino describió la importancia de la literatura latinoamericana para Italia en una entrevista de 1984. En esta ocasión, el autor italiano, importantísimo para el contexto de las traducciones de latinoamericanos al italiano debido a su papel de asesor editorial para Einaudi, afirmaba rotundamente que la oficialidad de las palabras de algunos autores latinoamericanos lo fastidiaba, porque detestaba que un escritor se volviera un personaje oficial y a los escritores involucrados en causas políticas prefería aquellos que sabían transmitir un espíritu internacional (Riccio 11). Pero aludiendo todavía más a las dinámicas editoriales que se dieron alrededor de la literatura latinoamericana Calvino admitía, de forma implícita, un desfase entre el campo cultural italiano e hispanoamericano cuando, con respecto a la traducción de la obra de Alejo Carpentier hecha por Longanesi en los años 50, afirmaba que: "Tante cose si pubblicavano ma hanno dovuto aspettare un altro momento" (Riccio 11$)^{2}$.

Aunque se trate de una opinión personal, las palabras de Calvino son muy interesantes por más de un motivo. En primer lugar, su “odio” a los escritores oficiales nos parece representativo de la opinión general que el campo cultural italiano mantuvo hacia la literatura latinoamericana a lo largo de cuatro décadas, entre 1950 y 1990. El estudio de los documentos de archivo editoriales hechos hasta ahora ${ }^{3}$ informa sobre las dificultades que el ámbito editorial italiano enfrentó para incluir en sus catálogos textos demasiado políticos o vinculados con una posición socio-política demasiado evidente, con lo que es posible imaginar cierto recelo hacia la producción literaria latinoamericana, sobre todo a raíz de los enfrentamientos, políticos y sociales, que caracterizaron los años 70 italianos. A lo largo de toda la segunda mitad del siglo XX la recepción editorial de la literatura latinoamericana vivió altibajos constantes: aunque se tradujeran muchísimos títulos el interés del campo cultural y literario se veía influido

1 Como lo explicaremos más adelante: no consideraremos las reseñas a las ediciones Rizzoli y Einaudi de la novela de Lezama Lima.

2 "Muchas cosas se publicaron pero tuvieron que esperar otro momento" [traducción mía].

3 Véase Carini, "Tra mediazione” y "Ciro Alegría”. 
por una lectura política y no literaria de las obras. En nuestra opinión esto causó el encubrimiento de muchas de las calidades literarias de los textos latinoamericanos y, al mismo tiempo, la imposición de estereotipos que facilitaban aquellos textos más fácilmente ubicables en un contexto determinado como lo fue, a partir de 1967 y de la aparición de Cien años de soledad, el del realismo mágico (Carini, “Etiquetas literarias”). De esta forma, la referencia hecha por Calvino a un desfase en el interés que caracterizó la mirada de los italianos hacia la literatura latinoamericana es, quizás, la admisión más sincera de los obstáculos que el campo editorial italiano encontró a la hora de interpretar y trasladar a otro contexto las obras procedentes de América Latina.

\section{2 ¿Por qué las reseñas?}

A partir de estos motivos pensamos que el análisis de las reseñas pueda representar un método adecuado para establecer qué esquemas interpretativos se utilizaron para leer las obras latinoamericanas y, al mismo tiempo, para empezar a tomar nota de cuánta distancia existía entre el campo cultural italiano y las obras latinoamericanas. Desde este punto de vista el análisis de la recepción a través de las reseñas mide las décimas de la acogida reservada a cada literatura en traducción y finalmente ayuda a identificar todos aquellos elementos que, ajenos a la literatura, contribuyeron a la formación del capital literario de los latinoamericanos en Italia (Casanova 31).

Por lo que se refiere a nuestro objeto de estudio, las primeras traducciones de Paradiso y Farabeuf, aparecieron en el mercado italiano en 1971 y fueron recibidas de forma muy distinta por la crítica.

Paradiso fue recibido de forma positiva, sobre todo en relación con la narración pseudo autobiográfica elegida por el autor, considerada estilísticamente óptima y, aunque complicada, fascinante y digna de interés literario. A los elogios se emparejaron algunas polémicas políticas, que sin embargo no perjudicaron el valor de la novela para el público italiano -hecho confirmado tanto por la gran cantidad de reseñas como por el alto número de ediciones que se produjeron a lo largo de los años. Al contrario, la centralidad del sexo y de la tortura en Farabeuf representaron un problema para los reseñistas, que tan solo le dedicaron una veintena de reseñas (incluyendo las entradillas), algunas de ellas totalmente negativas.

Una lectura más atenta del conjunto de reseñas disponibles permite dividirlas según varias tipologías:

- reseñas en revistas literarias,

- reseñas en periódicos, 
- entradillas,

- reseñas de escritores publicadas en periódicos.

Cada una de estas tipologías mantiene las características propias del medio en el que se publica, pero las reseñas en periódicos y las reseñas de escritores permiten una mayor profundización, ya que reflejan, por un lado, la ideología del periódico en el que aparecen y, por otro, el pulso del campo cultural visto desde un protagonista del ámbito creativo. Por consiguiente, es de particular interés el análisis de estas dos categorías, ya que permiten enfrentarse de forma más directa con las dinámicas -tanto políticas como literarias- que rodean el campo literario y que "influyen" en y "deciden” sobre el porvenir de un autor según esquemas jerárquicos predefinidos (Bourdieu 279).

\title{
3 Farabeuf en los años 70: un libro incómodo
}

Por lo que se refiere a Farabeuf, podemos identificar dos tipos de reseñas: las que valoran la novela dentro del conjunto de las obras latinoamericanas y las que valoran la eficacia de su temática. En relación con la primera categoría encontramos juicios positivos, como el de Ignazio Delogu, donde se distingue de forma neta la postura desde la que nacen Farabeuf y Cien años de soledad, la novela latinoamericana sin duda más conocida en el ámbito italiano en esos años. Delogu define el estilo de Elizondo como algo que diferente a las narraciones latinoamericanas más populares:

\begin{abstract}
lontan[o] dalla volontà di creare per comprendere, di forzare, sia pure, la realtà ma per impadronirsi della sua essenza proprie di un García Márquez o di un Vargas Llosa. Elizondo, con la sua dichiarata incapacità e impossibilità di dare un senso alla realtà e all'esperienza, sembra rappresentare l'altra faccia della medaglia, l'alternativa polare a un modo di vedere, di esistere, di raccontare ${ }^{4}$.
\end{abstract}

De la misma forma, el reseñista anónimo de Corriere Ticino identifica Farabeuf con la estética decadente europea y subraya la habilidad con la que Elizondo llega a elaborar su novela a partir de estímulos refinados que prevén una competencia formal elevada: “Elizondo rappresenta nell'ambito della letteratura

4 "Lejos de la voluntad de crear para comprender, de forzar, la realidad para apoderarse de su esencia propia de un García Márquez o de un Vargas Llosa. Elizondo, con su declarada incapacidad e imposibilidad para dar un sentido de realidad a la experiencia, parece representar la otra cara de la medalla, la alternativa polar a una forma de ver, de existir, de contar" [traducción mía]. 
latino-americana, la corrente che mira ad appropriarsi delle esperienze più raffinate della cultura europea in materia di técnica narrativa, di soluzione formale del linguaggio" ("Funambulismo intellettuale") 5 . Ambas reseñas reconocen el valor de la novela comparándola con la producción latinoamericana de la época e insertándolas en un contexto literario específico. Pero existe otro grupo de reseñas, de corte menos literario, que llega a considerar la novela amoral y negativa. En estas reseñas aparece, de forma bastante explítica, la voluntad de alejarse de los temas de Elizondo atribuyendo al libro, al autor y al tema tratado una valoración de tipo moral. En una entradilla de un autor anónimo Farabeuf es definido como "un romanzo macabro, oscuro e ossessivo. Negativo dal lato morale, blasfemo da quello religioso"6 ("Salvador Elizondo. Farabeuf"); en otra reseña es descrita como una "tromperie” literaria, "un vaneggiamento presuntuoso in cui si mescolano, come ingredienti di base, l'erotismo malato alle degeneranti crudezze"7 (Grigliè s.p.) sin que esto pueda tener alguna relación con los méritos del autor o con el papel que este libro puede llegar a tener dentro del ámbito literario. Podría parecer una contraposición bastante típica en referencia a una novela compleja y seguramente difícil, pero en el conjunto de reseñas que se produjeron sobre Farabeuf nos llama la atención la de Mario Luzi, publicada en las columnas del Corriere della Sera en el marco de la actividad como reseñista de latinoamericanos que el poeta italiano desempeñó de 1967 a 1974. Luzi, que a lo largo de sus reseñas traza un panorama bastante completo y coherente de la literatura latinoamericana, dedica a Elizondo una de sus reseñas en 1971; en ella señala los méritos del autor por su habilidad en la construcción formal de la obra, pero en el último párrafo de la reseña parece dar marcha atrás, por lo menos por lo que se refiere al tema, que juzga difícil de tolerar: "I luoghi comuni della letteratura "perversa” e dell'erotismo nero sono i più feroci che ci possano venire imposti. E certo, non basta questo raffinato prodotto di un discepolo di Bataille passato attraverso gli arcanes di André Breton, a farceli tollerare" ("Farabeuf")

5 "Elizondo representa en el ámbito de la literatura latinoamericana, la corriente que apunta a apoderarse de las experiencias más refinadas de la cultura europea en cuanto a la técnica narrativa, de solución formal del lenguaje" [traducción mía].

6 "Una novela macabra, oscura y obsesiva. Negativa desde el punto de vista moral, blasfema desde el religioso” [traducción mía].

7 "Un desvarío presumido en el que se mezclan, como ingredientes básicos, el erotismo enfermo a una depravada crudeza" [traducción mía].

8 "Los clichés de la literatura "perversa” y del erotismo negro son los más duros que puedan ser impuestos. Y por cierto, no basta este refinado producto de un discípulo de Bataille pasado por los arcanes de André Breton para que lo toleremos” [traducción mía]. 


\subsection{Paradiso en los años 70: entre política y literatura}

También en el caso de Lezama Lima es posible identificar dos diferentes categorías de reseñas: las literarias tout court y aquellas que además de lo literario incluyen comentarios políticos o referencias a los mecanismos del campo literario. Lo que más resalta es que para la mayoría de los reseñistas el estilo y la estructura de la novela del escritor cubano apelan a Proust, Joyce y Musil, y lo afirman con un consentimiento casi general. La novela del autor es considerada una obra maestra y la calidad de su literariedad se afianza reseña tras reseña. Pero alrededor de las reseñas a Paradiso surge un fenómeno peculiar, que es la apertura de brechas de debate cultural en las que se discuten dos elementos interesantes para nuestro análisis: la forma con la que la intelectualidad italiana lee las novelas latinoamericanas y su conocimiento de las mismas. En este sentido, una de las reseñas más polémicas es la de Giuseppe Bonura, que se publica bajo el título provocador "Rifugiati nella magia". El tema central es el cuestionamiento del interés hacia la literatura latinoamericana, que para Bonura reside en la similitud de temas y situaciones que son el espejo de lo que el lector italiano vive y ha vivido y no una simple fascinación política:

Può darsi, invece, che le ragioni siano da ricercarsi in altra sede, fuori dal contesto proprio della letteratura. Che siano, ad esempio, ragioni politiche. (Ma i critici qualunquisti ricordano con un sorrisetto ebete i fenomeni d'infatuazione per la narrativa russa e ungherese nell'Italia paleoindustriale, e poi la cotta per la narrativa nordamericana nel dopoguerra. Ebbene, anche in quei casi, l'infatuazione fu "anche" politica) [. . . Questi libri ci parlano di una realtà che, pur appartenendo a un mondo sviluppato, immobile, in cui non si muove foglia che la Cia non voglia [...] in qualche modo ci coinvolge. Anzi ci sembra addirittura emblematica di una situazione universale, planetaria: quella appunto dell'uomo preso tra la morsa del capitalismo colonizzatore perfino delle coscienze e la ribellione anarchica, da apocalisse. Tra la supina accettazione del sistema e l'avventura per l'avventura, è la seconda che dà luogo dal mito, o all'utopia, o al sogno: almeno presso i giovani'

\footnotetext{
9 "Puede ser, en cambio, que las razones tengamos que buscarlas en otra sede, fuera del contexto propio de la literatura. Que sean, por ejemplo, razones políticas. (Pero los críticos recuerdan con una sonrisita tonta los fenómenos de enamoramiento de la literatura rusa y húngara en la Italia paleoindustrial, y además el enamoramiento de la narrativa norteamericana en la posguerra. Pues bien, incluso en aquellos casos, el enamoramiento fue "también” político) [...] Estos libros nos hablan de una realidad que, no obstante pertenezca a un mundo desarrollado, inmóvil, en el que no se mueve hoja que la CIA no quiera [. . . ] de alguna forma nos comprometen. Es más, hasta nos parece emblemático de una situación universal, planetaria: aquella del hombre atrapado entre el capitalismo colonizador hasta en las conciencias y la rebelión anárquica, de apocalipsis. Entre la aceptación del sistema y la aventura por la aventura, es la segunda que da lugar al mito, a la utopía, o al sueño: por lo menos entre los jóvenes” [traducción mía].
} 
La referencia no puede ser que una politización de la recepción de la literatura latinoamericana -debido también a la acción, totalmente política, de editores como Feltrinelli- que pudo caracterizar el mercado editorial italiano de los años 70. De hecho, en aquellos años la cultura italiana empezó a escindirse en una confrontación entre cultura baja y alta/cultura revolucionaria y conservadora (Ferretti) que inevitablemente recaía también en la recepción de los productos editoriales. Pero analizando el extracto de la reseña de Bonura percibimos que su cuestionamiento no es solo el resultado de una problematización política, sino que tiene que ver también con la disponibilidad que el campo cultural demuestra hacia esta literatura. En particular, nos interesa la primera parte de la cita, porque ahí Bonura parece hacer referencia a cierto esnobismo que circulaba en los ambientes editoriales y literarios italianos y que iba en descrédito de las obras procedentes de América Latina. El reproche a una pasión literaria que se ve impulsada por la política sugiere que la calidad de la literatura latinoamericana se viera anulada por su vinculación con la política y la reivindicación. Por esto no nos sorprende que, a unos meses de salir Paradiso, Dario Puccini escribiera una reseña que más allá de los contenidos literarios sobre la obra nos interesa por las referencias implícitas a las dinámicas extraliterarias que caracterizaban el ámbito literario italiano.

En primer lugar, Puccini propone una lectura de Paradiso que resalte sus peculiaridades estéticas más allá de la comparación con cualquier autor europeo, oponiéndose, de hecho, al emparejamiento con Musil, Proust y otros que venía haciéndose desde su llegada a las librerías:

quando si sono fatti i nomi di Proust o di Joyce o di Musil, aggiungendo per Lezama Lima soltanto l'aggettivo "tropicale" per poter in qualche modo spiegare la natura conturbante e frenetica di questo viaggio nella memoria [...] si sono soltanto tracciate alcune linee generiche della genealogia di Paradiso: la cui sostanza è un gongorismo a misura oceanica e a fermentazione tropicale [. . . ] la letterarietà di Paradiso è qualcosa di diverso dalla letterarietà nostra, occidentale. Mentre la seconda, infatti, sembra non possa andar disgiunta da concetti come gusto, misura, squisitezza, finitezza, scelta impeccabile delle parole e degli stilemi, la prima appare caotica, smisurata, automatica, visionaria (giustamente si è detto che Paradiso va ricollegato alle forme del tardo surrealismo), e in qualche modo spontanea ed elementare ${ }^{10}$. ("Il Paradiso di Lezama Lima")

10 "Cuando se hicieron los nombres de Proust o de Joyce o de Musil, añadiendo para Lezama Lima solo el adjetivo "tropical” para poder de alguna manera explicar la naturaleza perturbadora y frenética de este viaje en la memoria [. . . s se trazaron solo algunas líneas genéricas de la genealogía de Paradiso, cuya sustancia es un gongorismo oceánico con fermentación tropical [. . . ] la literariedad de Parasido es algo distinto de la literariedad nuestra, occidental. Mientras la segunda, en efecto, parece no poder ir separada de conceptos como gusto, mesura, exquisitez, finitud, elección impecable de las palabras y de los símbolos, la primera parece 
Puccini, que fue uno de los primeros hispanistas italianos en el siglo XX, subraya la necesidad de referirse a Lezama Lima utilizando referencias menos europeas y sí más tradicionalmente relacionadas con la literatura latinoamericana- para conseguir una comparación más adecuada tanto desde el punto de vista estilístico como del temático. En efecto, los autores que propone como referencias son Melville, Faulkner, el primer Neruda, el primer Asturias, Guimarães Rosa y el García Márquez de Cien años de soledad, más en línea con el punto de vista del que comienza el relato autobiográfico. Puccini, además, distingue de forma evidente el origen de la literariedad latinoamericana, dirigiéndose de forma implícita a aquellos que dentro del campo cultural se obstinaban a leer a las obras procedentes de América Latina simplificando y juzgando el texto desde un punto de vista "europeo". Lo mismo haría Luzi desde el Corriere della Sera: sin entrar de algún modo en el tema político cuestionaría la inexactitud de las primeras asociaciones "europeas" con Proust, Musil y Joyce, reivindicando la alteridad de la producción literaria latinoamericana como característica peculiar y valiosa:

La demiurgia di Proust, di Musil e di Joyce nasce da una intuizione primaria sulla natura profonda dell'uomo ma è anche ordinata intorno a un criterio, per così dire, formante. In questi maestri del romanzo europeo moderno la profondità è la dimensione in cui vive l'eroe dissociato dalla normalità del mondo, ma è anche l'origine di un sistema implicito di conoscenza e di relazione con il mondo. [.. .] Sebbene questi autori siano stati tutti invocati per dare una parentela a Lezama Lima, non vedo niente di tutto questo in Paradiso. In primo luogo non c'è qui un retroterra di crisi e di frustrazione, ma siamo anzi nel pieno di un atteggiamento salutare e sapienziale. La tendenza ad ammettere a coinvolgere il mondo nella sua totalità di tempi e di modi non obbedisce a un movimento di recessione vorticosa della coscienza, ma a un naturale movimento di espansione e di conquista verso un'ultima possibile completezza ${ }^{11}$. ("Paradiso")

caótica, desmesurada, automática, visionaria (con razón se ha dicho que Paradiso hay que relacionarlo a las formas del surrealismo tardío), en alguna forma espontánea y elemental” [traducción mía].

11 "La demiurgía de Proust, de Musil y de Joyce nace de una intuición primaria sobre la naturaleza profunda del hombre, pero se ordena alrededor de un criterio, digamos así, que forma. En estos maestros de la novela europea moderna la profundización es la dimensión en la que vive el héroe disociado de la normalidad del mundo, pero es también el origen de un sistema implícito de conocimientos y de relaciones con el mundo. [...] No obstante, estos autores se invoquen para dar familia a Lezama Lima, no veo nada de todo esto en Paradiso. En primer lugar, aquí no hay todo ese bagaje de crisis y frustración, al contrario, estamos en el medio de una actitud saludable y sapiencial. La tendencia a incluir el mundo en su totalidad de tiempos y modos no obedece a un movimiento de recesión de la conciencia, sino a un natural movimiento de expansión y de conquista hacia una última posible situación completa" [traducción mía]. 
Sin embargo, es en el cierre donde Puccini se enfrenta con la cuestión del valor de los latinoamericanos y de cómo leerlos, delatando, una vez más, la distancia que separaba el ámbito italiano del latinoamericano. Es así como en la frase de cierre de su reseña Puccini añade una clara referencia a las múltiples alusiones a la política y a la supuesta cercanía de Lezama Lima que se manifiestan en las reseñas tras el caso Padilla y concluye -aunque con trece años de antelación con respecto a Calvino- señalando la lejanía del campo cultural italiano:

non è senza significato che le due opere maggiori della letteratura cubana contemporanea, Il secolo dei lumi di Alejo Carpentier e Paradiso di Lezama Lima, si riferiscano a un passato remoto e coloniale. La cosa non ci scandalizza, ma neppure ci esalta (vogliamo parlare del caso Padilla?). Del resto personalmente non ho il minimo dubbio (e l'ho in parte dimostrato) che Paradiso sia una opera d'alta civiltà letteraria, e un vero capolavoro della letteratura dell'America Latina. Volutamente lasciando in sospeso ogni altro discorso è questo che qui conta per ora affermare ${ }^{12}$. ("Il Paradiso di Lezama Lima")

Esta última consideración nos llama la atención porque se empareja con una muy similar hecha por Mario Luzi en ocasión de la salida de Raccattacadaveri en 1969:

Il nostro taccuino di appunti latino-americani si infittisce rapidamente di nomi nuovi o che arrivano in Italia per la prima volta. Forse l'ho già scritto, ma è bene ribattere che non si tratta di un fuoco d'artificio editoriale: la letteratura del continente si trova davvero in un periodo di manifesto rigoglio. Non tento neppure di spiegarlo il perché, sapendo quanto il rapporto di causa ed effetto sia aleatorio se applicato a questo argomento. È vero che il forte di alcuni tipi di critica -non più soltanto dello storicismo- è appunto di fornire quelle spiegazioni; ma il fenomeno ricorrente delle stagioni creative rimane, tutto sommato, inesplicabile e perentorio. Mi limito così ad osservare che la letteratura latinoamericana usufruisce di una condizione unica (non del tutto invidiabile, certo, ma indubbiamente vitale) determinata soprattutto dal coesistere di questi fattori: l'aprirsi e l'insorgere della coscienza alla tragedia sociale e politica del continente, la scoperta simultanea della realtà e del mito autoctoni, il trapianto vigoroso di tecniche artistiche europee e nord americane. Per ultimo il più prezioso: l'integrità non corrosa dei sentimenti dell'uomo ${ }^{13}$. ("L'asceta del peccato")

12 "No es sin significado que las dos más grandes obras cubanas contemporáneas, Il secolo dei lumi de Alejo Carpentier y Paradiso de Lezama Lima, se refieran a un pasado remoto y colonial. La cosa no nos indigna, pero tampoco nos exalta (¿queremos hablar del caso Padilla?). En resumidas cuentas, personalmente no tengo la menor duda (y en parte lo demostré) de que Paradiso es una obra de alta civilización literaria, y una verdadera obra maestra de la literatura de América Latina. Dejando cualquier otro discurso a un lado adrede, es esto lo que importa aquî” [traducción mía].

13 "Nuestro cuaderno de apuntes latinoamericano se hace rápidamente más denso de nombres nuevos que llegan a Italia por primera vez. Quizás ya lo escribí, pero es bueno recordar que no se trata de fuegos artificiales de la edición: la literatura del continente se encuentra realmente en un momento de manifiesta plenitud. No intenta ni siquiera explicar el porqué, 
Como es posible deducir, el valor literario de la literatura latinoamericana es reiterado una y otra vez. Esto puede ocurrir porque esta literatura frecuentemente iba acompañada por una ideologización que se relacionaba con el contexto extra literario, pero, de hecho, pensamos que estas frecuentes declaraciones sirvieran como justificación ante esa parte del circuito cultural que no tenía una visión de conjunto lo suficientemente amplia como para comprender el aspecto multifacético de la propuesta literaria procedente del subcontinente. por esto la interpretaba aplicando estereotipos (Carini "Tra mediazione”, "Etiquetas literarias"). Es aquí, entonces, donde podemos empezar a comprender la importancia de una reflexión sobre las consecuencias que los elementos extraliterarios pudieron tener sobre la difusión de la literatura latinoamericana en traducción.

Un grupo de reseñas aluden de forma más precisa a la relación que el autor y la obra tuvieron con el régimen castrista, posiblemente para contextualizar su procedencia y, pensamos, para insertarlos en un contexto editorial y literario específicos dentro del ámbito cultural italiano. Lo que sí se percibe es cierto cuestionamiento sobre la validez de la aproximación apolítica de Lezama Lima después del caso Padilla, pero sin llegar a construir un caso político alrededor del texto literario.

\section{Las re-traducciones en el siglo XXI}

La situación respecto a la segunda ola de reseñas que decidimos analizar es totalmente diferente. Ante todo, es bastante diferente la situación de llegada de estas nuevas ediciones. Mientras Farabeuf no vuelve a ser reeditado hasta 2018, Paradiso lo es en tres diferentes ocasiones entre 1971 y 2016. En 1990, por la editorial Rizzoli, con una traducción que pasa de estar al cargo de Storchi y Riva a ser solo de Valerio Riva, de quien resultan también las notas. En 1992,

sabiendo cuánto la relación entre causa y efecto pueda ser aleatoria si se aplica a este argumento. Es verdad que lo fuerte de cierta crítica - no solo del historicismo es el de dotar aquellas explicaciones, pero el fenómeno cíclico de las estaciones de la creatividad humana, al final, inexplicables y perentorias. Me limito de este modo a observar que la literatura latinoamericana goza de una condición única (no envidiable, por cierto, pero indudablemente vital), determinada sobre todo por el coexistir de estos factores: el abrirse y el insurgir de la conciencia de la tragedia social y política del continente, el descubrimiento simultáneo de la realidad y del mito autóctono, el implante vigoroso de técnicas artísticas europeas y norteamericanas. Por último el más valioso: la integridad no corroída de los sentimientos del hombre" [traducción mía]. 
para la editorial Einaudi, con una re-traducción a cargo de Glauco Felici, a la que se añaden un conjunto de epitextos que complementan la lectura: nota del traductor y un apéndice con trascripciones, glosario de voces cubanas e hispánicas, un repertorio de lugares, cosas y personajes y un apartado titulado "Coordenandas lezamianas" con notas que explican la genealogía de algunos personajes de la novela. En 2001, siempre por la editorial Einaudi, en una edición en la que es añadida una introducción a cargo de Mario Vargas Llosa.

Las ediciones a las que haremos referencia nosotros salen al mercado en noviembre 2016, para Edizioni SUR, y en 2018, para la editorial LiberAria. En la nueva edición de Paradiso se señalan algunos cambios: la traducción es la de Felici, pero se ha reinsertado el texto de Julio Cortázar que había acompañado la primera edición il Saggiatore y un prefacio de Chiara Valerio, escritora y editora italiana y, en 2016, directora cultural de la feria del libro de Milán Tempo di Libri. Farabeuf, en cambio, apareció en marzo de 2018 con una nueva traducción firmada por Giulia Zavagna y una introducción firmada por Alessandro Raveggi. Básicamente, la mayor novedad de las reediciones que interesaron a estas dos novelas es la anexión de nuevos paratextos. Algunos de estos paratextos (Valerio, Raveggi) son guías de lectura actualizadas a la contemporaneidad, en las que protagonistas del panorama literario italiano se dibujan discursos alrededor de la literatura latinoamericana. En otros casos, como los son el de Riccio y Vargas Llosa en las ediciones de 1991 y 2001, los paratextos tienen una faceta más latinoamericana, siendo escritos por una catedrática y un escritor que delinean la figura de Lezama Lima directamente desde un punto de vista latinoamericano.

Por lo que concierne las reseñas a las ediciones más recientes de las novelas -que son las que utilizaremos para este estudio- tenemos que señalar ante todo el formato diferente que las reseñas han adquirido en la práctica editorial. Las reseñas actuales no son simplemente un comentario, sino que se han vuelto verdaderos instrumentos de marketing que en muchas ocasiones funcionan en paralelo, y en acuerdo, con las estrategias editoriales. El primer dato que tenemos a nuestro alcance es que las reseñas son, para ambas novelas, escasas y carecen de una figura predominante en el escenario actual de la comunicación sobre libros: los bookbloggers no aparecen, lo que implica que siendo SUR y LiberAria editoriales independientes que cuentan con una red de distribución propia, la distribución de estas dos novelas se ha estructurado según los mecanismos tradicionales editorial-librería-lector, editorial-revista-lector, sin poder gozar de esa comunicación inmediata y directa que es posible a través de la participación hecha por internet a través de las redes sociales. Las reseñas a ambos libros cuentan con firmas de cierto nivel en el panorama literario actual: tanto Stefano Gallerani, para Paradiso, como Gianni Montieri, para 
Farabeuf, son dos personajes importantes y reconocidos en el ámbito literario italiano. Por lo que se refiere a Paradiso Gallerani actualiza las referencias culturales que se habían indicado en los años 70, añadiendo Pynchon y Roussel a los modelos que pueden ser útiles para acercarse a Lezama Lima. La reseña, que aparece en La Stampa, es a la vez una presentación del autor, una contextualización del mismo y una descripción del argumento de la obra, en la que nos parece faltar, sin embargo, una valoración personal por parte del autor con respecto a la forma de la obra. Más amplia y detenida en el análisis y la explicación de la estructura y de los significados de la obra es otra reseña a Paradiso, la de Francesca Lazzarato aparecida en la revista Alias. Lazzarato, traductora del español, presenta el contexto, el autor y el tema y comenta la estructura utilizando, cuando es necesario, también citas de autoridad -en este caso, Cortázar y Sarduy- que le permiten enmarcar la obra en un ámbito más específico, pero igualmente cercano a los lectores.

Por lo que se refiere a Farabeuf, en cambio, hasta este momento -junio 2018- las reseñas son solo cuatro, de las que solo una, la de Gianni Montieri, llega a profundizar sobre la novela con cierta fluidez. En su texto, publicado en el blog cultural Minima \& Moralia, se detiene en el valor de la memoria como fil rouge para la lectura del texto sin hacer alguna referencia al ámbito latinoamericano, es decir, interpretando la novela a partir de una experiencia totalmente personal y subjetiva de poeta. De hecho, pensamos que la literariedad de ambas novelas ha podido ser un límite en la comunicación directa e instantánea que se necesita en las redes sociales y que hoy predomina en el mundo del marketing editorial. Es quizás por esto que ciertos temas, como la memoria, el renacer, el sexo y, más en general, el replegarse en sí mismo que caracteriza ambas novelas pasa desapercibido y no es transmitido a los lectores italianos a través de estos epitextos. A nuestro parecer la complejidad -y la variedad- que caracterizan el ámbito literario latinoamericano siguen siendo difíciles de resumir y explicar. Este dato nos parece confirmado por la reseña que Goffredo Fofi dedicó a Paradiso en las páginas de la revista Internazionale: "Torna un classico del novecento poco frequentato dall'italica cultura" ${ }^{14}$, lo que confirma -nuevamente- la existencia de cierta dificultad en apoderarse de textos complejos o diferentes a los traducidos de costumbre dentro del marco del exotismo.

14 "Vuelve un clásico de 1900 poco frecuentado por la cultura italiana” [traducción mía]. 


\section{Conclusiones}

En conclusión, los resultados de nuestra investigación convergen hacia algunos elementos que nos parecen de suma importancia para definir la necesidad de ampliar los estudios de recepción, añadiendo a una interpretación estrictamente literaria de los epitextos una investigación a nivel cultural y editorial. Más allá de la valoración de los contenidos de una obra las palabras utilizadas por los reseñistas ayudan a comprender el tono y la predisposición con los que el ámbito cultural y literario está decidido a acoger una obra y un autor, lo que va a determinar su éxito o su fracaso. El análisis de los casos de Paradiso y Farabeuf, por ejemplo, permite individuar un problema común, el de una "literariedad latinoamericana", que impide una recepción "profesional" amplia. A esto añadimos que las reseñas de Bonura, Puccini y Luzi, ahí donde subrayan y distinguen la procedencia y el valor de la literariedad latinoamericana, apelan a un cambio de perspectiva en la mirada de aquellos profesionales y lectores que quieren acercarse a las obras procedentes de América Latina sin tomar nota de cuáles son sus características propias. Por otro lado, las reseñas de Bonura, Puccini y Luzi delatan la frustración que los tres sufren al tener que compartir el espacio literario con interpretaciones políticas sobre las obras latinoamericanas. Esto nos hace pensar que, en realidad, dentro del ámbito cultural italiano el interés en los latinoamericanos se concreta sobre todo en términos de contenidos. Se repite, entonces, la condición que se ha observado en los estudios de archivos sobre la mediación editorial: una porción del campo literario italiano tenía un escaso conocimiento de las características literarias latinoamericanas e interpretó sus productos a través de los estereotipos de la política y del exotismo.

A la hora de valorar la contribución de la literatura latinoamericana al campo literario italiano tendremos que tomar nota de estos elementos aunque no concuerden con la percepción que el número de títulos traducidos puede dar de la difusión de la literatura procedente de América Latina en Italia. Gracias a los resultados conseguidos, podemos afirmar la necesidad de ponderar la valoración de la recepción de las obras latinoamericanas considerando tanto su traducción -con datos numéricos y estadísticas- como los métodos y las dinámicas que se relacionan a esta ${ }^{15}$. En general, para comprender la real

15 Puccini hace referencia a cierto problema de comprensión entre ámbito literario italiano y literatura latinoamericana también en una reseña a Il grande Sertao y Paradiso, publicada por Puccini en la revista Avanti el 25 de julio de 1971: "I più importanti libri latino-americani di questa annata letteraria, tradotti in italiano sono stati Grande Sertao di Joao Guimaraes Rosa (ed. Feltrinelli) e Paradiso di José Lezama Lima (ed. Il Saggiatore). Del primo si è parlato poco, 
configuración de la recepción de la literatura latinoamericana en Italia y, más adelante, anotar cuáles fueron las repercusiones que tuvo sobre la literatura italiana, es necesario considerar cuáles autores han sido traducidos y leídos y cuáles no. Por esto habrá que valorar su inserción o no inserción en el campo cultural desde una perspectiva múltiple, que se proponga comprender qué imagen de América Latina se proporcionó a los lectores y cuáles dinámicas (políticas, económicas, culturales y literarias) la argumentaba. Esto implica que nos preguntemos, por ejemplo, hasta qué punto pudieron comprender la literatura latinoamericana ciertos reseñistas si no conocían la totalidad de la oferta procedente de América Latina. Del mismo modo, es oportuno preguntarse si se puede juzgar o trabajar sobre la literatura latinoamericana prescindiendo de los autores telúricos o políticos, así como si la que se ha traducido ha sido un fiel reflejo de la producción literaria latinoamericana o si tan solo se ha llegado a conocer una pequeña parte de ella. Este enfoque interesa en primer lugar para una profundización del estudio del ámbito literario italiano, pero, en vista de una comunicación más amplia y rápida de los textos, también para el estudio del ámbito literario hispanoamericano y, aún más, de las relaciones entre ámbitos editoriales y de sus consecuentes repercusiones a nivel de producción literaria por parte de autores cuanto más conectados y en relación entre sí incluso desde espacios físicos diferentes.

\section{Bibliografía}

Anónimo. “Funambolismo intelletuale'. Reseña de Farabeuf, de S. Elizondo”. Corriere del Ticino, 27 de marzo 1971.

--.. “'Salvador Elizondo. Farabeuf'. Reseña de Farabeuf, de S. Elizondo”. Libri di ieri e di oggi, enero 1971.

ed è un vero peccato. Del secondo si è parlato molto, ma - a mio modo di vedere - con un'ottica ancora inadeguata. Non credo sia il caso di entrare qui in polemica con la critica europea (non solo italiana), che a tutti i costi ha cercato grandi riferimenti nella letteratura occidentale (ed es. Joyce e Proust) per penetrare nel mondo allucinante e labirintico ed allegorico, di Paradiso. Se a tutt'oggi rimangono misteriosi (anche perché poco noti) fenomeni europei come il barocco di Góngora e di Quevedo, tanto più rimangono misteriosi i loro 'figli' americani, da Sor Juana Inés de la Cruz (contemporanea di Calderón) a José Lezama Lima (contemporaneo si fa per dire - di Carlo Emilio Gadda). Del resto, per scoprire e riconoscere fenomeni che nella letteratura d'oltre oceano appaiono abbastanza "europei” come Borges e Cortázar ci è voluto parecchio tempo, e lo aiuto della pubblicità e del cinema (Blow Up). Per scoprire e riconoscere Lezama Lima e Guimaraes Rosa ci vorrà ancor più tempo" ("Paradiso"). 
Bonura, Giuseppe. “'Rifugiati nella magia’. Reseña de Paradiso, de J. Lezama Lima”. Sette giorni, 23 de mayo 1971, s.p.

Bourdieu, Pierre. Le regole dell'arte. Genesi e struttura del campo letterario. il Saggiatore, 2013 [1992].

Cadioli, Alberto. Le diverse pagine. il Saggiatore, 2012.

Carini, Sara. "Etiquetas literarias y cánones distorsionados: la literatura latinoamericana en Italia entre estereotipos y miradas desenfocadas”. Centroamericana, 26.1, 2016, pp. 27-43.

---. “Ciro Alegría en la 'Medusa' Mondadori: éxitos y problemas de una mediación editorial”, América Latina y la literatura mundial. Mercado editorial, redes globales y la invención de un continente. Eds. Gesine Müller y Dunia Gras. Iberoamericana/Vervuert, 2015, pp. 143-162.

--.. "Tra mediazione e incomprensione: la ricezione editoriale e le letterature straniere. Il caso delle Meduse latinoamericane in Mondadori". Letteratura e archivi editoriali. Nuovi spunti d'autore. Le carte d'archivio strumento di critica letteraria. Eds. Carmela Pierini, Sara Carini, Elisa Bolchi. Aracne editrice, 2014, pp. 75-103.

Casanova, Pascale. La República mundial de las Letras. Anagrama, 2001 [1999].

Chartier, Roger. L'ordine dei libri. il Saggiatore, 1994 [1992].

Delogu, Ignazio. "Salvador Elizondo. Farabeuf”. Rinascita, 1 de enero de 1971, s.p.

Ferretti, Gian Carlo. Il mercado delle lettere. Industria cultural e lavoro critico in Italia dagli anni cinquanta ad oggi. Einaudi, 1979.

Fofi, Goffredo. “'Un classico cubano da riscoprire’. Reseña de Paradiso, de J. Lezama Lima”. Internazionale, 2 de diciembre 2016, p. 100.

Gallerani, Stefano. “'Lezama Lima, il Paradiso dello scandalo’. Reseña de Paradiso, de J. Lezama Lima”. Il Mattino, 30 de diciembre 2016, p. 19.

Grigliè, Remo [Grig. R]. “'Oh che bel piacere la chirurgia erotica!'. Reseña de Farabeuf, de

S. Elizondo". Stampa sera, 2 de octubre 1970.

Lazzarato, Francesca. “'Lezama Lima, una carezza sul caos’. Reseña de Paradiso, de J. Lezama Lima”. Alias, Il Manifesto, 24 de noviembre 2016, p. 1.

Luzi, Mario. “'Paradiso'. Reseña de Paradiso, de J. Lezama Lima”. Corriere letterario, Corriere della Sera, 13 de mayo 1971, p. 12.

---. “'Farabeuf'. Reseña de Farabeuf, de S. Elizondo". Corriere letterario, Corriere della Sera, 24 de diciembre 1970, p. 12.

--.. “'L'asceta del peccato. Reseña de Raccattacadaveri, de J.C. Onetti”. Corriere letterario, Corriere della Sera, 9 de noviembre 1969, p. 13.

Montieri, Gianni. “'Più Tenace della memoria. Farabeuf di Salvador Elizondo'. Reseña de Farabeuf, de S. Elizondo". Minima \& Moralia, 16 de mayo 2018. http://www.minimaetmo ralia.it/wp/memoria-farabeuf-salvador-elizondo/, consultado 3 de junio 2018.

Puccini, Dario. “'Il “Paradiso” di Lezama Lima'. Reseña de Paradiso, de J. Lezama Lima”.

Paese Sera, 18 de junio 1971.

-.-. "Paradiso". Avanti. 25 de julio 1971.

Riccio, Alessandra. “'Scrittori esemplari io vi odio tutti'. Entrevista a Italo Calvino”. l'Unità, 20 de septiembre 1984.

Ricœur, Paul. Tempo e racconto, vol III. Jaka Book, 1988. 